\title{
A Snapshot of a Homogeneous Spinning Universe
}

\author{
Barbaro Quintero-Leyva \\ Independent Work, Miami FL, USA \\ Email: doserate2002@yahoo.com
}

How to cite this paper: Quintero-Leyva, B. (2018) A Snapshot of a Homogeneous Spinning Universe. Open Access Library Journal, 5: e4895.

https://doi.org/10.4236/oalib.1104895

Received: September 10, 2018

Accepted: September 26, 2018

Published: September 29, 2018

Copyright $\odot 2018$ by author and Open Access Library Inc.

This work is licensed under the Creative Commons Attribution International License (CC BY 4.0).

http://creativecommons.org/licenses/by/4.0/

\begin{abstract}
A snapshot of the circular speed as a function of the radius in a spinning-homogeneous spherical universe was obtained using a mass-dependent characteristic-acceleration in the Modified Newtonian Dynamics (MOND paradigm as a modified $2^{\text {nd }}$ law of Newton) with and without considering the impact of the relativistic speed. To consider the impact of the relativistic speed the Extended Newtonian Theory (ENET), previously developed by the author, was used. The corresponding kinetic energy equation for ENET is however reported in this work for the first time. The speed profile shows a non-linear trend with features that has been experimentally noted before. It was shown that the Hubble law (for circular speeds) can be inferred from the results for a distance range close to the experimental results of the Hubble telescope key project. The calculation considering the impact of the relativistic speeds yields a very distinctive tail towards the edge of the universe that has been noted before. It is striking that a spinning universe model yields (without any reference to dark matter or dark energy) observed features of a universe which, based on photometric and spectral line measurements, is currently interpreted as radially expanding at an accelerated rate.
\end{abstract}

\section{Subject Areas}

Classical Mechanics, Classical Physics, Modern Physics

\section{Keywords}

Celestial Mechanics, Newtonian Gravitation, Newton's $2^{\text {nd }}$ Law, Theory of Relativity, Perihelion Precession, Cosmology

\section{Introduction}

In 1929 Hubble [1] found a linear correlation between galaxies' receding veloci- 
ties and distance; he emphasized that the linear relation "is a first approximation representing a restricted range in distance". The same year Zwicky [2] published an analysis covering the finding of Hubble and assessed the potential processes behind the spectral shifts representing the apparent velocities of recession of the galaxies. Among the potential explanation for the observations he makes detail remarks on the gravitational drag of light and developed a corresponding model for preliminary calculations. In 1998 Riess et al. [3] found evidences for an interpretation of an accelerating universe based on measurement of photometric and spectral data of 10 type Ia supernovae with redshift between 0.16 and 0.62 . These results are the reason for the frequently mentioned concept of dark energy.

In 1933 Zwick [4], to explain the observed discrepancy between the velocity dispersion measured in the Coma cluster and the velocity dispersion obtained from the virial theorem (using the luminosity mass), inferred as one possibility the presence of dark matter. In the 1970s the need for dark matter in the outer part of galaxies and well beyond their optical edge was reported [5] and references there in].

A rotating universe could be an alternative to a radially expanding universe. Ideas in this direction could be dated back to the 1940's [6] [7] [8] and references therein].

Note that it is widely believed (as stated frequently in the scientific literature) that the receding velocities of the galaxies are the result of an expanding space and the existence of a vacuum energy and that as mentioned before the discrepancy between measured velocities and the calculated ones based on Newtonian theory is due to the presence of dark matter. These interpretations are not assumed in the present work.

In this work a snapshot of the circular speed as a function of the radius in a spinning-homogeneous spherical universe was obtained using a mass dependent-characteristic acceleration in the MOND paradigm (applied on the $2^{\text {nd }}$ law of Newton) with and without considering the impact of the relativistic speed. The impact of the relativistic speed was considered using the Extended Newtonian Theory previously developed by the author. The corresponding kinetic energy equation for that theory is presented here.

The rest of this work is structured as follows: Section 2 makes an introduction to the concept of critical density from energy and force point of view. In section 3 a mass dependent-characteristic acceleration equation is fitted to acceleration data obtained from binned experimental data set of galaxies and galaxy clusters. That correlation is used to determine the circular speed as a function of distance using the so extended MOND paradigm (HYB model) without considering the impact of the relativistic speeds. In section 4 the circular speed calculation is made by modifying the HYB model with the equation of ENET to consider the impact of the relativistic speeds. In section 5 remarks are made related to the results and methods of previous sections. Section 6 shows the summary and the 
concluding remarks.

\section{On the Concept of Critical Density}

The Newtonian energy balance of a self-gravitating universe can approximately be written as

$$
E=T+U=\frac{1}{2} M \dot{R}^{2}-\frac{4}{3} \pi G \rho \cdot R^{2} M
$$

where

$\mathrm{T}$ : kinetic energy

$U \approx-\frac{G M^{2}}{R}:$ Potential energy

$G$ : Newton's gravitational constant

$M$ : Mass of the observable universe

$R, \dot{R}$ : Radius to the edge of the observable universe and its time derivative

$\rho$ : Volumetric mass density of the universe (assumed to be a constant in large scale)

Defining a critical density as the one which makes $E=0$ (static universe!) implies

$$
\rho_{c}=\frac{3}{8 \pi G} \frac{\dot{R}^{2}}{R^{2}}
$$

Assuming that $\dot{R}$ and $R$ could be correlated with the Hubble law for galaxies, the critical density can be written as

$$
\rho_{c}=\frac{3 H_{0}^{2}}{8 \pi G} \approx 9.7 \times 10^{-30} \mathrm{~g} / \mathrm{cm}^{3} \text { for } H_{0}=2.32 \times 10^{-18} \mathrm{~s}^{-1}
$$

This equation is frequently found in the literature.

Note that in order for $E$ (in Equation (1)) to be zero in a radially expanding universe $\dot{R}$ cannot be zero so the universe cannot be static therefore contradicting the assumption used to obtain $\rho_{c}$. However for a rotating universe (where the cosmic structures are moving with elliptical orbits for example) the contradiction disappears.

The baryonic mass density (based on the observed and predicted abundance of light element formed in the early universe) is about $\rho_{b} \approx 2 \times 10^{-31} \mathrm{~g} / \mathrm{cm}^{3}$ which is about two orders of magnitude smaller than the critical density. The current postulate of the non-baryonic dark matter and dark energy is frequently based on this discrepancy.

A different equation can be obtained from the balance between the Newtonian gravitational force and Newton's $2^{\text {nd }}$ law:

$$
\frac{G \frac{4}{3} \pi \cdot R^{3} \rho M}{R^{2}}=M a_{0} \quad a_{0} \text { : Apparent radial acceleration of the universe (azi- }
$$
muthal acceleration for a rotating universe)

$$
\text { If } a_{0}=c H_{0}, \Rightarrow \rho_{c}=\frac{3 c H_{0}}{4 \pi G R}=\frac{3 H_{0}^{2}}{4 \pi G} \approx 1.9 \times 10^{-29} \mathrm{~g} / \mathrm{cm}^{3} \quad \text { which is about }
$$


twice the value based on energy balance.

Note also that using the definition of a self-gravitating sphere is [9] $U=-\int_{0}^{R} \frac{G V \mathrm{~d} m}{r}$ ( $V$ is the volume and $\mathrm{d} m$ is the mass differential) the critical density based on energy balance is $\rho_{c}=\frac{5 H_{0}^{2}}{8 \pi G} \approx 1.6 \times 10^{-29} \mathrm{~g} / \mathrm{cm}^{3}$ which is closer to the force-balance value.

\section{A Spinning Universe Neglecting the Impact of Relativistic Speeds}

A snapshot (circular speed as a function of the radius) of a spinning spherical universe can be obtained by solving the equation obtained in [10] (2017a):

$$
\mathrm{v}^{4}-G M \frac{\mathrm{v}^{2}}{r}-G M a_{0}(M)=0
$$

In that reference it was hypothesized that the characteristic acceleration of MOND [11] was not a constant and that it is a function of the mass of the system in question (e.g. galaxy, galaxy clusters, super-clusters, universe). It was also reported there that a function of the form $a_{0}(M)=k_{0}+k_{1} M+k_{2} \sqrt{M}$ (M: mass in units of the mass of the universe, $\sum k_{i} \approx a_{0}\left(M_{U}\right)$,

$k_{0} \approx a_{0 \mathrm{MOND}} \approx a_{0}\left(M_{U}\right) / 6$ ) fits adequately the values of the acceleration calculated from the binned experimental data published in [12]. That equation however does not go monotonously from $k_{0}$ to $a_{0}\left(M_{U}\right)$. The following function (Asymmetrical Sigmoidal)

$$
a_{0}(M)=k_{0}+\frac{k_{1}-k_{0}}{\left(1+\left(\frac{M}{k_{2}}\right)^{k_{3}}\right)^{k_{4}}}
$$

however fits adequately the acceleration values and does go monotonously from $k_{1}\left(\approx a_{0 \mathrm{MOND}}\right)$ to $k_{0}\left(a_{0}\left(M_{U}\right)\right)$. The parameters are: $k_{0}=6.972813 \times 10^{-10}$, $k_{1}=1.791984 \times 10^{-10}, k_{2}=8.819503 \times 10^{-11}, k_{3}=1.150579$, $k_{4}=4.626816 \times 10^{-01}$. These parameters are the result of an empirical modification of the initial fit of the regression performed in [13]. The initial values of $k_{0}, \cdots, k_{4}$ were multiplied by factors of $1.365,1.5,220,2$ and 2 respectively (see Figure 1 and Table 1 for details).

Note in Equations (2) and (3)that $M$ is a function of the radius and it extends from magnitudes corresponding to galaxies to magnitudes comparable to the whole visible universe. For a given mass, the corresponding radius is determined assuming a constant volumetric mass density of the universe so $r=\left(\frac{3 M}{4 \pi \rho}\right)^{1 / 3}$.

Even though Equation (2) can be solved exactly in terms of its coefficients, it was solved iteratively (for a better comparison with the results of the next section) isolating the speed in the quartic term. For verification purpose Equation (2) was also solved by evaluation every $1 \mathrm{~m} / \mathrm{s}$ in search for sign changes. 


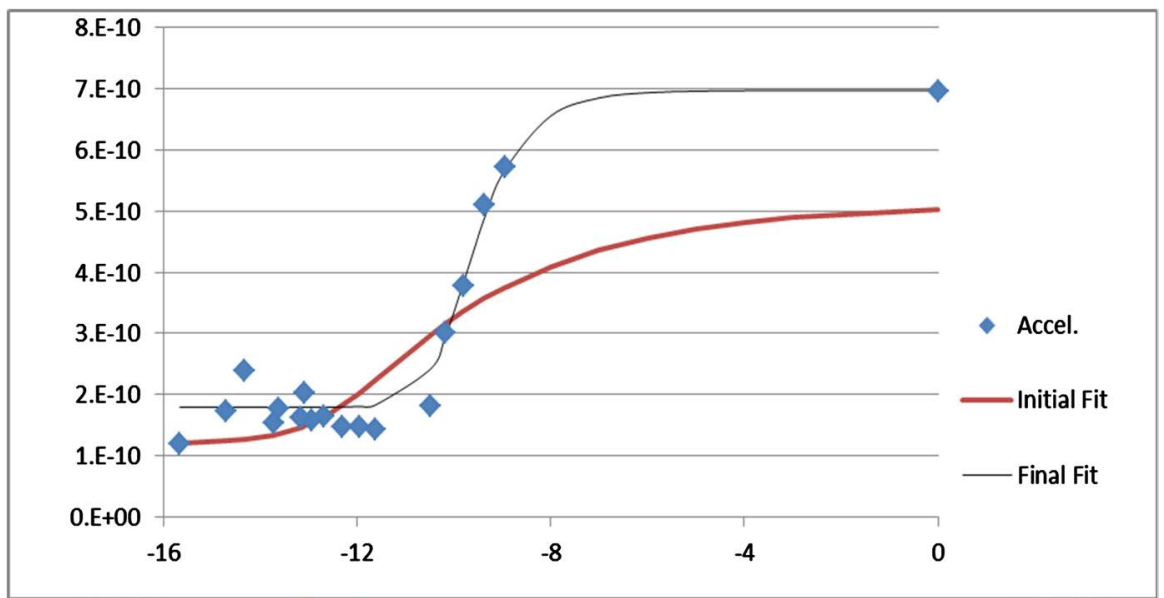

Figure 1. Fits to the acceleration $\left(\mathrm{m} / \mathrm{s}^{2}\right)$ vs. $\log 10$ of mass (relative to the mass of the universe).

Table 1. Fits to the circular acceleration vs. mass data.

\begin{tabular}{|c|c|c|c|}
\hline $\begin{array}{c}\mathrm{M} \text { ass } \\
\text { (Univ. ratio) }\end{array}$ & $\begin{array}{l}\text { Accel. } \\
\mathrm{m} / \sec ^{2}\end{array}$ & Initial Fit & Final Fit \\
\hline 1.00 & $6.97 \mathrm{E}-10$ & $5.02 \mathrm{E}-10$ & $6.97 \mathrm{E}-10$ \\
\hline $1.00 \mathrm{E}-01$ & & $4.99 \mathrm{E}-10$ & $6.97 \mathrm{E}-10$ \\
\hline $1.00 \mathrm{E}-02$ & & $4.95 \mathrm{E}-10$ & $6.97 \mathrm{E}-10$ \\
\hline $1.00 \mathrm{E}-03$ & & $4.89 \mathrm{E}-10$ & $6.97 \mathrm{E}-10$ \\
\hline $1.00 \mathrm{E}-04$ & & $4.81 \mathrm{E}-10$ & $6.97 \mathrm{E}-10$ \\
\hline $1.00 \mathrm{E}-05$ & & $4.70 \mathrm{E}-10$ & $6.96 \mathrm{E}-10$ \\
\hline $1.00 \mathrm{E}-06$ & & $4.56 \mathrm{E}-10$ & $6.94 \mathrm{E}-10$ \\
\hline $1.00 \mathrm{E}-07$ & & $4.36 \mathrm{E}-10$ & $6.85 \mathrm{E}-10$ \\
\hline $1.00 \mathrm{E}-08$ & & $4.09 \mathrm{E}-10$ & $6.56 \mathrm{E}-10$ \\
\hline $1.14 \mathrm{E}-09$ & $5.72 \mathrm{E}-10$ & $3.75 \mathrm{E}-10$ & $5.68 \mathrm{E}-10$ \\
\hline $4.25 \mathrm{E}-10$ & $5.10 \mathrm{E}-10$ & $3.57 \mathrm{E}-10$ & $4.88 \mathrm{E}-10$ \\
\hline $1.58 \mathrm{E}-10$ & $3.78 \mathrm{E}-10$ & $3.35 \mathrm{E}-10$ & $3.84 \mathrm{E}-10$ \\
\hline $6.58 \mathrm{E}-11$ & $3.00 \mathrm{E}-10$ & $3.15 \mathrm{E}-10$ & $2.94 \mathrm{E}-10$ \\
\hline $3.30 \mathrm{E}-11$ & $1.81 \mathrm{E}-10$ & $2.97 \mathrm{E}-10$ & $2.42 \mathrm{E}-10$ \\
\hline $2.39 \mathrm{E}-12$ & $1.44 \mathrm{E}-10$ & $2.23 \mathrm{E}-10$ & $1.83 \mathrm{E}-10$ \\
\hline $1.12 \mathrm{E}-12$ & $1.47 \mathrm{E}-10$ & $2.03 \mathrm{E}-10$ & $1.81 \mathrm{E}-10$ \\
\hline $4.88 \mathrm{E}-13$ & $1.47 \mathrm{E}-10$ & $1.82 \mathrm{E}-10$ & $1.80 \mathrm{E}-10$ \\
\hline $2.08 \mathrm{E}-13$ & $1.65 \mathrm{E}-10$ & $1.64 \mathrm{E}-10$ & $1.79 \mathrm{E}-10$ \\
\hline $1.14 \mathrm{E}-13$ & $1.57 \mathrm{E}-10$ & $1.54 \mathrm{E}-10$ & $1.79 \mathrm{E}-10$ \\
\hline $8.10 \mathrm{E}-14$ & $2.03 \mathrm{E}-10$ & $1.49 \mathrm{E}-10$ & $1.79 \mathrm{E}-10$ \\
\hline $7.05 \mathrm{E}-14$ & $1.61 \mathrm{E}-10$ & $1.47 \mathrm{E}-10$ & $1.79 \mathrm{E}-10$ \\
\hline $2.34 \mathrm{E}-14$ & $1.77 \mathrm{E}-10$ & $1.35 \mathrm{E}-10$ & $1.79 \mathrm{E}-10$ \\
\hline $1.86 \mathrm{E}-14$ & $1.54 \mathrm{E}-10$ & $1.33 \mathrm{E}-10$ & $1.79 \mathrm{E}-10$ \\
\hline $4.77 \mathrm{E}-15$ & $2.38 \mathrm{E}-10$ & $1.26 \mathrm{E}-10$ & $1.79 \mathrm{E}-10$ \\
\hline $1.99 \mathrm{E}-15$ & $1.73 \mathrm{E}-10$ & $1.24 \mathrm{E}-10$ & $1.79 \mathrm{E}-10$ \\
\hline $2.18 \mathrm{E}-16$ & $1.19 \mathrm{E}-10$ & $1.21 \mathrm{E}-10$ & $1.79 \mathrm{E}-10$ \\
\hline
\end{tabular}


The mass was considered an input expanding from $2 \mathrm{E} 11$ to $2.45 \mathrm{E} 21$ solar masses, the baryonic mass density used was $\sim 1.1 \mathrm{E}-28 \mathrm{~kg} / \mathrm{m}^{3}\left(\sim 1.1 \mathrm{E}-31 \mathrm{~g} / \mathrm{cm}^{3}\right)$.

Figure 2 (see Table 2 also) shows the circular speed calculated from Equation (2) as a function radius (from 9.52E22 $\mathrm{m}(\sim 3 \mathrm{Mpc})$ to $2.192 \mathrm{E} 26 \mathrm{~m}(\sim 7087 \mathrm{Mpc})$ ) for the Kepler model $\left(a_{0}=0, \mathrm{v}=\sqrt{G M(r) / r}\right)$, for MOND model $\left(a_{0} \approx c H_{0} / 6\right)$ and for the HYB model ( $a_{0}$ calculated from Equation (3)). The upper curve is to be discussed in the next section. From that figure can be seen that the speed profile of the MOND model is significantly greater (about twice as large at the edge) than the Kepler model. The HYB model speed profile is even larger (about 3 times as large at the edge). Notice that in terms of the classical-kinetic energy the ratios are even larger (about 4 and 9 for MOND and HYB respectively) because of the quadratic dependence on the speed. It is noted that experimental confirmation of non-linear redshift-distance law has been reported before (see for example ref [14]). Note also that the Kepler model yields a true linear relation (as expected) between circular speed and distance but the slope is more than 10 times smaller than $H_{0}$.

The degree of the polynomial in each model of Figure 2 was determined based on the minimum degree that yields a coefficient of determination $\left(\mathrm{R}^{2}\right)$ equal or greater than 0.998 and that visually touches at least the last 10 points with a monotonous behavior. Note that the coefficient of the linear term of the HYB model is close to $H_{0}$, which suggests that a linear model for some region of the curve could be consistent with the Hubble law. In fact a linear fit between $\sim 50 \mathrm{Mpc}$ and $\sim 500 \mathrm{Mpc}$ yields $H_{0} \approx 1.0 \mathrm{E}-18 \mathrm{~s}^{-1}$ with $\mathrm{R}^{2}=0.995$. Similar results could be derived directly from eq. 2 by neglecting the term containing $\frac{v^{2}}{r}: \quad v=\left(\frac{4}{3} \pi G \rho a_{0}\right)^{1 / 4} r^{3 / 4}$ which when it is developed in Taylor series (up to the linear term) around $r_{0}$, the slope can be written as $s=\frac{3}{4}\left(\frac{4}{3 r_{0}} \pi G \rho a_{0}\right)^{1 / 4}$. For $r_{0}=10 \mathrm{Mpc} \Rightarrow s \approx 2.2 \mathrm{E}-18 \mathrm{~s}^{-1}$. For $r_{0}=50 \mathrm{Mpc} \Rightarrow s \approx 1.5 \mathrm{E}-18 \mathrm{~s}^{-1}$. It is noted that for the slope calculation the value of $a_{0}$ was assumed to be $6.97 \mathrm{E}-10$ for both values of $r_{0}$. This assumption does not significantly impact the results because $a_{0}$ does not change much in this range.

The results just discussed shows that the Hubble law can be inferred from the HYB model using only a characteristic acceleration and a proper distance range.

\section{A Spinning Universe Considering the Impact of Relativistic Speeds}

It is noted from Figure 2 that at least the last 8 points representing the HYB model involve relativistic speeds. To consider the impact of the relativistic speed on the inertial acceleration, ref [15] modified the Newtonian acceleration in a way that it correctly yields the intrinsic (relativistic) perihelion precession of the planets (e.g. 43 “/cy for Mercury). The acceleration was modified by applying two consecutive boosts of time dilation and length contraction. This modification 
Table 2. Circular speed vs. distance (m).

\begin{tabular}{|c|c|c|c|c|}
\hline \multirow{2}{*}{$\mathrm{r}$} & \multicolumn{4}{|c|}{$\mathrm{v}(\mathrm{m} / \mathrm{s})$} \\
\hline & Kepler & Mond & Hyb & Enet-Hyb \\
\hline $9.52 \mathrm{E}+22$ & $1.67 \mathrm{E}+04$ & $2.36 \mathrm{E}+05$ & $3.02 \mathrm{E}+05$ & $3.02 \mathrm{E}+05$ \\
\hline $1.20 \mathrm{E}+23$ & $2.10 \mathrm{E}+04$ & $2.81 \mathrm{E}+05$ & $3.79 \mathrm{E}+05$ & $3.79 \mathrm{E}+05$ \\
\hline $1.51 \mathrm{E}+23$ & $2.65 \mathrm{E}+04$ & $3.34 \mathrm{E}+05$ & $4.71 \mathrm{E}+05$ & $4.71 \mathrm{E}+05$ \\
\hline $1.90 \mathrm{E}+23$ & $3.34 \mathrm{E}+04$ & $3.97 \mathrm{E}+05$ & $5.79 \mathrm{E}+05$ & $5.79 \mathrm{E}+05$ \\
\hline $2.40 \mathrm{E}+23$ & $4.21 \mathrm{E}+04$ & $4.72 \mathrm{E}+05$ & $7.04 \mathrm{E}+05$ & $7.04 \mathrm{E}+05$ \\
\hline $3.02 \mathrm{E}+23$ & $5.30 \mathrm{E}+04$ & $5.62 \mathrm{E}+05$ & $8.50 \mathrm{E}+05$ & $8.50 \mathrm{E}+05$ \\
\hline $3.81 \mathrm{E}+23$ & $6.68 \mathrm{E}+04$ & $6.68 \mathrm{E}+05$ & $1.02 \mathrm{E}+06$ & $1.02 \mathrm{E}+06$ \\
\hline $4.80 \mathrm{E}+23$ & $8.41 \mathrm{E}+04$ & $7.95 \mathrm{E}+05$ & $1.22 \mathrm{E}+06$ & $1.22 \mathrm{E}+06$ \\
\hline $6.05 \mathrm{E}+23$ & $1.06 \mathrm{E}+05$ & $9.46 \mathrm{E}+05$ & $1.46 \mathrm{E}+06$ & $1.46 \mathrm{E}+06$ \\
\hline $7.62 \mathrm{E}+23$ & $1.34 \mathrm{E}+05$ & $1.13 \mathrm{E}+06$ & $1.74 \mathrm{E}+06$ & $1.74 \mathrm{E}+06$ \\
\hline $9.60 \mathrm{E}+23$ & $1.68 \mathrm{E}+05$ & $1.34 \mathrm{E}+06$ & $2.08 \mathrm{E}+06$ & $2.08 \mathrm{E}+06$ \\
\hline $1.21 \mathrm{E}+24$ & $2.12 \mathrm{E}+05$ & $1.59 \mathrm{E}+06$ & $2.48 \mathrm{E}+06$ & $2.48 \mathrm{E}+06$ \\
\hline $1.52 \mathrm{E}+24$ & $2.67 \mathrm{E}+05$ & $1.90 \mathrm{E}+06$ & $2.95 \mathrm{E}+06$ & $2.95 \mathrm{E}+06$ \\
\hline $1.92 \mathrm{E}+24$ & $3.37 \mathrm{E}+05$ & $2.26 \mathrm{E}+06$ & $3.51 \mathrm{E}+06$ & $3.51 \mathrm{E}+06$ \\
\hline $2.42 \mathrm{E}+24$ & $4.24 \mathrm{E}+05$ & $2.68 \mathrm{E}+06$ & $4.18 \mathrm{E}+06$ & $4.18 \mathrm{E}+06$ \\
\hline $3.05 \mathrm{E}+24$ & $5.34 \mathrm{E}+05$ & $3.19 \mathrm{E}+06$ & $4.97 \mathrm{E}+06$ & $4.97 \mathrm{E}+06$ \\
\hline $3.84 \mathrm{E}+24$ & $6.73 \mathrm{E}+05$ & $3.80 \mathrm{E}+06$ & $5.92 \mathrm{E}+06$ & $5.92 \mathrm{E}+06$ \\
\hline $4.84 \mathrm{E}+24$ & $8.48 \mathrm{E}+05$ & $4.53 \mathrm{E}+06$ & $7.04 \mathrm{E}+06$ & $7.04 \mathrm{E}+06$ \\
\hline $6.09 \mathrm{E}+24$ & $1.07 \mathrm{E}+06$ & $5.39 \mathrm{E}+06$ & $8.38 \mathrm{E}+06$ & $8.38 \mathrm{E}+06$ \\
\hline $7.68 \mathrm{E}+24$ & $1.35 \mathrm{E}+06$ & $6.42 \mathrm{E}+06$ & $9.97 \mathrm{E}+06$ & $9.98 \mathrm{E}+06$ \\
\hline $9.67 \mathrm{E}+24$ & $1.70 \mathrm{E}+06$ & $7.64 \mathrm{E}+06$ & $1.19 \mathrm{E}+07$ & $1.19 \mathrm{E}+07$ \\
\hline $1.22 \mathrm{E}+25$ & $2.14 \mathrm{E}+06$ & $9.10 \mathrm{E}+06$ & $1.41 \mathrm{E}+07$ & $1.41 \mathrm{E}+07$ \\
\hline $1.54 \mathrm{E}+25$ & $2.69 \mathrm{E}+06$ & $1.08 \mathrm{E}+07$ & $1.68 \mathrm{E}+07$ & $1.68 \mathrm{E}+07$ \\
\hline $1.93 \mathrm{E}+25$ & $3.39 \mathrm{E}+06$ & $1.29 \mathrm{E}+07$ & $2.00 \mathrm{E}+07$ & $2.01 \mathrm{E}+07$ \\
\hline $2.44 \mathrm{E}+25$ & $4.27 \mathrm{E}+06$ & $1.54 \mathrm{E}+07$ & $2.38 \mathrm{E}+07$ & $2.39 \mathrm{E}+07$ \\
\hline $3.07 \mathrm{E}+25$ & $5.38 \mathrm{E}+06$ & $1.84 \mathrm{E}+07$ & $2.83 \mathrm{E}+07$ & $2.85 \mathrm{E}+07$ \\
\hline $3.87 \mathrm{E}+25$ & $6.78 \mathrm{E}+06$ & $2.19 \mathrm{E}+07$ & $3.37 \mathrm{E}+07$ & $3.41 \mathrm{E}+07$ \\
\hline $4.88 \mathrm{E}+25$ & $8.55 \mathrm{E}+06$ & $2.61 \mathrm{E}+07$ & $4.02 \mathrm{E}+07$ & $4.07 \mathrm{E}+07$ \\
\hline $6.14 \mathrm{E}+25$ & $1.08 \mathrm{E}+07$ & $3.12 \mathrm{E}+07$ & $4.78 \mathrm{E}+07$ & $4.88 \mathrm{E}+07$ \\
\hline $7.74 \mathrm{E}+25$ & $1.36 \mathrm{E}+07$ & $3.72 \mathrm{E}+07$ & $5.70 \mathrm{E}+07$ & $5.87 \mathrm{E}+07$ \\
\hline $9.75 \mathrm{E}+25$ & $1.71 \mathrm{E}+07$ & $4.44 \mathrm{E}+07$ & $6.79 \mathrm{E}+07$ & $7.10 \mathrm{E}+07$ \\
\hline $1.23 \mathrm{E}+26$ & $2.15 \mathrm{E}+07$ & $5.31 \mathrm{E}+07$ & $8.09 \mathrm{E}+07$ & $8.65 \mathrm{E}+07$ \\
\hline $1.55 \mathrm{E}+26$ & $2.71 \mathrm{E}+07$ & $6.35 \mathrm{E}+07$ & $9.64 \mathrm{E}+07$ & $1.07 \mathrm{E}+08$ \\
\hline $1.95 \mathrm{E}+26$ & $3.42 \mathrm{E}+07$ & $7.60 \mathrm{E}+07$ & $1.15 \mathrm{E}+08$ & $1.39 \mathrm{E}+08$ \\
\hline $2.19 \mathrm{E}+26$ & $3.85 \mathrm{E}+07$ & $8.33 \mathrm{E}+07$ & $1.26 \mathrm{E}+08$ & $1.79 \mathrm{E}+08$ \\
\hline
\end{tabular}




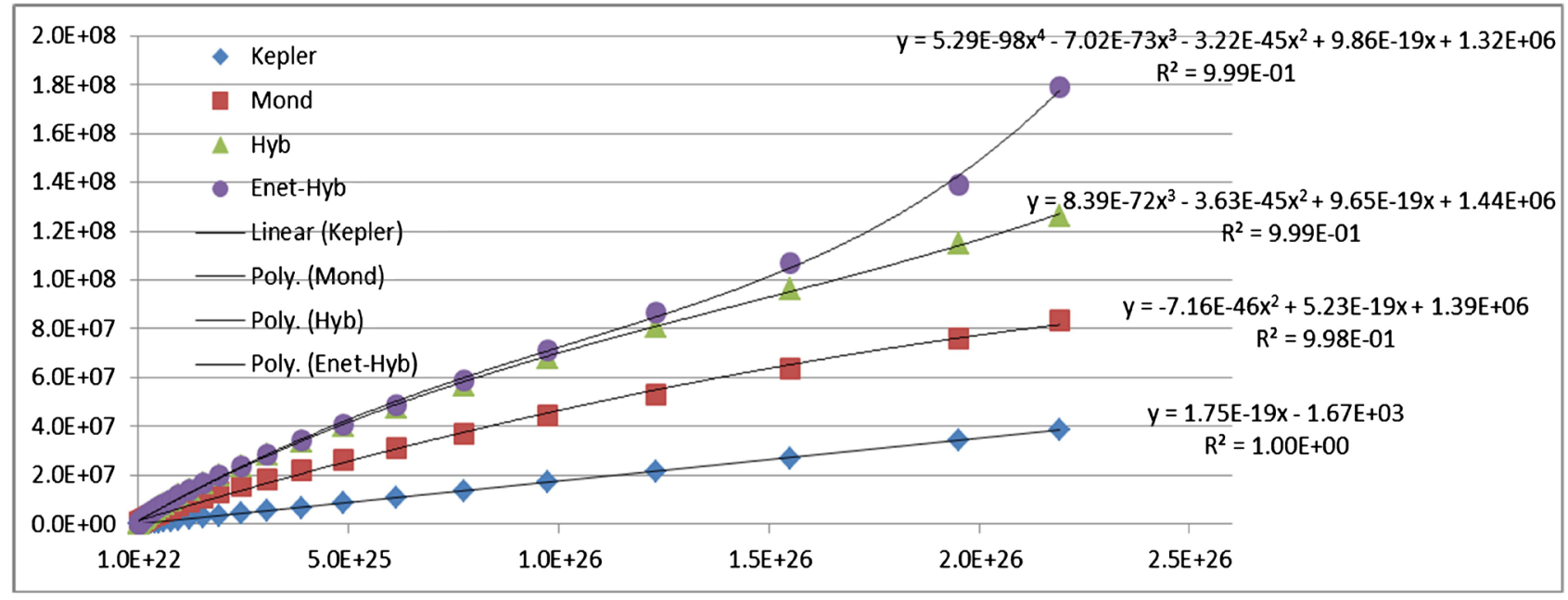

Figure 2. Circular speed (m/s) vs. distance $(\mathrm{m})$.

is called ENET (Extended NEwtonian Theory). Ref. [10] (2017b) applied that correction to the HYB model (Equation (2)) and obtained:

$$
-\frac{1}{c^{6}} \mathrm{v}^{10}+\frac{3}{c^{4}} \mathrm{v}^{8}-\frac{3}{c^{2}} \mathrm{v}^{6}+\mathrm{v}^{4}-G M \frac{\mathrm{v}^{2}}{r}-G M a_{0}=0
$$

It is emphasized that Equation (4) is the result of three modifications (assuming the establishment of a circular acceleration) to the $2^{\text {nd }}$ law of Newton when it is equated to Newton's gravitational law: 1 ) MOND model (as a modified $2^{\text {nd }}$ law of Newton); 2) HYB model (use of a mass dependent $a_{0}$ (Equation (3)) in MOND); 3) ENET model (application of two consecutive boosts to the classical concept of time and space interval in the Newtonian concept of inertial acceleration).

Equation (4) was solved iteratively by isolating the speed in the quartic term and by straight forward evaluation for verification. The results of the calculations are shown in Figure 2 (see also Table 2). From that figure can be seen that from about 8E25 m ( 2505 Mpc) the ENET-HYB model (Equation (4)) starts significantly deviating (increasing) with respect to the HYB model, at the top of the tail the speed is about 4.6 the speed yielded by the KEPLER model (the square of the ratio of the speeds is about 21.6).

It is noted that significant non-linear speed-distance law (the tail in Figure 2) for very large distances has been reported before and alternative fundaments for this behavior have also been proposed (see for example Ref. [16] and references therein).

\section{Additional Remarks}

It is remarkable that Equation (4), which neither assumes a radially accelerating universe (dark energy) nor dark matter, yields many features of the universe as:

- The Baryonic Tully-Fisher (BTF) law for spiral galaxies (asymptotically flat rotation curve: $\mathrm{v} \ll c$ and $\left.\frac{\mathrm{v}^{2}}{r} \ll a_{0}\right), a_{0}$ could effectively be considered a 
statistical constant (from rotation curve fits) or a galaxy-dependent parameter from the galaxy's surface gravity [10] (2017b).

- The BTF for galaxy clusters ( $\mathrm{v} \ll c$ and $\frac{\mathrm{v}^{2}}{r} \ll a_{0}, a_{0}$ could effectively be determined from Equation (3)).

- The Hubble law ( $\mathrm{v} \ll c, \frac{\mathrm{v}^{2}}{r} \ll a_{0}$, and $r_{0}$ of the order of 10 to $500 \mathrm{Mpc}, \mathrm{a}_{0}$ could effectively be determined from Equation (3)). Note that if the inferred (from photometric and spectral line measurements) velocity (magnitude and direction) as function of distance is not accurate enough there could be room for a circular speed interpretation (or a mixture of circular and radial speed) instead of just a radial speed interpretation (radial accelerating expansion or just radial expansion).

- The very steep non-linear speed-distance relation (the tail in Figure 2) towards the edge of the universe.

Perhaps the concept of critical density should not be the figure of merit to address the dark matter/energy problem. Even the use of the kinetic energy as a figure of merit could be dubious considering that the derivation of the kinetic energy from $E_{k}=\int_{0}^{r} F f_{r}(v) \mathrm{d} r$ with $F=m f_{a}(\mathrm{v}) \frac{\mathrm{d} v}{\mathrm{~d} t}$ could yield very different numerical results and trends for speeds comparable to the speed of light. For example,

for Newtonian acceleration $\left(f_{a}(\mathrm{v})=f_{r}(r)=1\right) \quad E_{k}=\frac{1}{2} m \mathrm{v}^{2}$, for Einstein's STR $\left(f_{a}(\mathrm{v})=\left(1-\frac{\mathrm{v}^{2}}{c^{2}}\right)^{-1}, f_{r}(\mathrm{v})=\left(1-\frac{\mathrm{v}^{2}}{c^{2}}\right)^{-1 / 2}\right)$ $E_{k}=m c^{2}\left[\left(1-\frac{\mathrm{v}^{2}}{c^{2}}\right)^{-1 / 2}-1\right]$,

for $\operatorname{ENET}\left(f_{a}(\mathrm{v})=\left(1-\frac{\mathrm{v}^{2}}{c^{2}}\right)^{3}, \quad f_{r}(\mathrm{v})=\left(1-\frac{\mathrm{v}^{2}}{c^{2}}\right)^{1 / 2}\right) E_{k}=\frac{1}{9} m c^{2}\left[1-\left(1-\frac{\mathrm{v}^{2}}{c^{2}}\right)^{9 / 2}\right]$.

The direct measurement of the Newtonian velocities and/or acceleration could perhaps be a better figure of merit to assess the predictability of a theory if the measurements' uncertainties are sufficiently small to differentiate between different theories.

\section{Summary and Concluding Remarks.}

A snapshot of the circular speed as a function of the radius in a spinning-homogeneous spherical universe was obtained using a variable characteristic acceleration (mass dependent) in the MOND paradigm (applied on the $2^{\text {nd }}$ law of Newton) with and without considering the impact of the relativistic speed. To consider the impact of the relativistic speed the Extended Newtonian Theory, previously developed by the author, was used. The corresponding kinetic energy 
equation for that theory was however reported in this work for the first time.

The speed profile shows a non-linear trend with some features that has been experimentally noted before. It was shown that the Hubble law (in a circular speed context) can be inferred (with fits or Taylor series) from the results for a distance range close to the experimental results of the Hubble telescope key project. The calculation considering the impact of the relativistic speeds yields a very distinctive tail towards the edge of the universe that has also been noted before.

It is remarkable that a spinning universe model yields many observed features of a universe which, based on measurements, is currently interpreted as radially expanding at an accelerated rate.

It could be worthy to measure, as far as possible, the correlation of mass vs. radius (no assumption of constant mass density) of rotationally linked cosmic structures to see, for example, if the characteristic acceleration can be determined from the corresponding surface gravity.

\section{Acknowledgements}

I would like to thank the anonymous reviewer for comments on the presentation of this work

\section{Conflicts of Interest}

The authors declare no conflicts of interest regarding the publication of this paper.

\section{References}

[1] Hubble, E. (1929) A Relation between Distance and Radial Velocity among Extragalactic Nebulae. Proceedings of the National Academy of Sciences of the United States of America, 15, 168-173. Astronomy. https://doi.org/10.1073/pnas.15.3.168

[2] Zwicky, F. (1929) On the Red Shift of Spectral Line through Interstellar Space. Proceedings of the National Academy of Sciences of the United States of America, 15, 773-779. Physics. https://doi.org/10.1073/pnas.15.10.773

[3] Riess, et al. (1998) Observational Evidence from Supernovae for an Accelerating Universe and a Cosmological Constant. arXiv:astro-ph/9805201v.

[4] Zwicky, F. (1933) The Redshift of Extragalactic Nebulae. Republication. Springer Science + Business Media LLC 2008.

[5] Bertone, G. and Hooper, D. (2016) A History of Dark Matter. FERMILAB-PUB-16157-A (astro-ph.CO. arXiv:1605.04909v2).

[6] Zorba, S. (2012) Dark Energy and Dark Matter as Inertial Effects. arXiv:1210.3021. [physics.gen-ph]

[7] Berman, M.S. (2013) Realization of Einstein's Machian Program. arXiv:1302.2498v1 [physics-gen-ph]

[8] Berman, M.S. and Gomide, F. (2013) Local and Global Stability of the Universe. Journal of Modern Physics, 4, 7-9. https://doi.org/10.4236/jmp.2013.48A002

[9] (2018) Scienceworld.wolfram.com 
[10] Quintero-Leyva, B. (2017a) On the Extension of the Baryonic Tully-Fisher Relation to Galaxy Clusters and Super Massive-Cosmic Systems. Open Access Library Journal, 4, e3686.

(2017b) On the Characteristic Acceleration of MOND. Open Access Library Journal, 4, e3576.

[11] Milgrom, M. (1983a) A Modification of the Newtonian Dynamics as a Possible Alternative to the Hidden Mass Hypothesis. Astrophysical Journal, 270, 365-370.

(1983b) A Modification of the Newtonian Dynamics. Implications for Galaxies. $A s$ trophysical Journal, 270, 371-389.

[12] McGaugh, S.S., et al. (2010) The Baryon Content of Cosmic Structures. The Astrophysical Journal, 708, L14-L17. https://doi.org/10.1088/2041-8205/708/1/L14

[13] (2018) MyCurveFit.com

[14] Segal, I.E. and Nicoll, J.F. (1992) Apparent Nonlinearity of the Redshift-Distance Relation in Infrared Astronomical Satellite Galaxy Samples. Proceedings of the National Academy of Sciences of the United States of America, 89, 11669-11672. Astronomy. https://doi.org/10.1073/pnas.89.24.11669

[15] Quintero-Leyva, B. (2016) An Extended Newtonian Theory for Gravitational Bound Systems. Open Access Library Journal, 3, e2678.

[16] Ashmore, L. (2006) Recoil between Photons and Electrons Leading to the Hubble Constant and CMB. Galilean Electrodynamics, 17, SI No. 3. 